\title{
Graphene Hybrids: Functionalized Nanomaterial for Single-step Immunoassay Based on Fluorescence Quenching and Size Separation
}

\author{
A. Shirai ${ }^{1}$, K. Sueyoshi', T. Endo ${ }^{1}$, H. Hisamoto ${ }^{1}$ \\ 1 Osaka Prefecture University, 1-1 Gakuen-cho, Naka-ku, Sakai-shi, Osaka, 599-8531, Japan, \\ hisamoto@chem.osakafu-u.ac.jp
}

\begin{abstract}
:
This paper reports the preparation of the polyethylene glycol-coated graphene nanoplatelet (PEG-GN) possessing highly dispersive, fluorescence quenching and size separation functions, and its application to "single-step" immunoassay. PEG-GN allowed to separate free fluorescently-labeled antibody and immunocomplex by the difference in size. The former adsorbed on PEG-GN and its fluorescence was quenched, and the latter did not approach the surface of PEG-GN due to the steric hindrance of PEG structure. By mixing PEG-GN and fluorescently-labeled antibody and sample solution containing antigen, the antigen concentration was able to be measured from the fluorescence intensity changes without washing operation. In this study, as a demonstration, the C-reactive protein (CRP) concentration was successfully measured by single-step operation.
\end{abstract}

Key words: Graphene, Immunoassay, Point of care testing (POCT), Single-step operation.

\section{Introduction}

In the field of medical diagnosis, immunoassay is very important method to measure the specific protein concentration. However, the conventional immunoassay is needed to be conducted on sophisticated instruments by trained medical professions due to the step-bystep operations. In order to eliminate those, we paid attention to graphene that has high fluorescence quenching function. In our previous studies, a capillary-type immunoassay microdevice with the soluble PEG coating containing graphene-antibody conjugates and fluorescently-labeled antibody achieved rapid measurement based on homogeneous reaction [1]. However, since the fluorescence quenching efficiency depends on the distance between fluorescent dye and graphene, this principle has underlying limitation about sensitivity. Thus, we conceive the principle by using the graphenecontaining hydrogel sheet. This material allowed to separate free fluorescently-labeled antibody and immunocomplex. The former was selectively adsorbed on the graphene surface in the hydrogel and was quenched. This principle suggested to solve the problem of sensitivity [2]. However, the response time was long because of the heterogeneous principle.
In this study, we prepared PEG-GN possessing highly dispersive, fluorescence quenching and size separation functions. As a demonstration, by mixing sample solution containing CRP, antiCRP antibody-FITC and GN-PEG, the CRP concentration was successfully measured with single-step operation (Scheme.1).

\section{Experimental}

At first, into a 200-mL round bottom flask, 50 $\mathrm{mg}$ of $\mathrm{GN}$, various weights of PEG (50-250 mg), $10 \mathrm{~mL}$ of 1-methyl-2-pyrrolidone, $20 \mathrm{~mL}$ of deionized water, and 10 drops of tributyl phosphate were added. Then, under vigorous

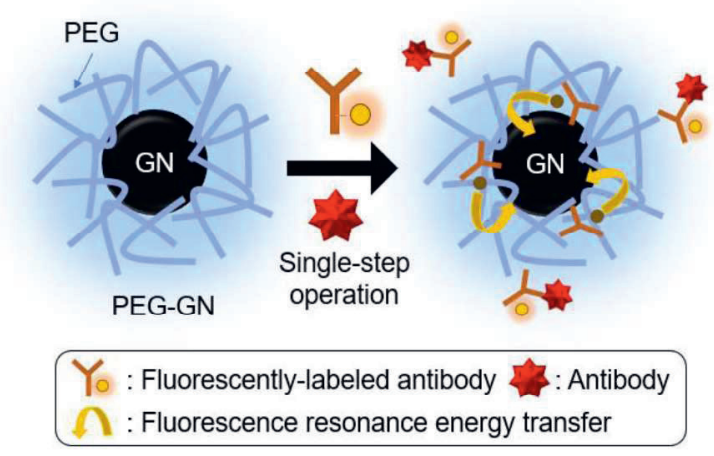

Scheme. 1. Immunoassay principle based on the multiple functions of PEG-GN. 


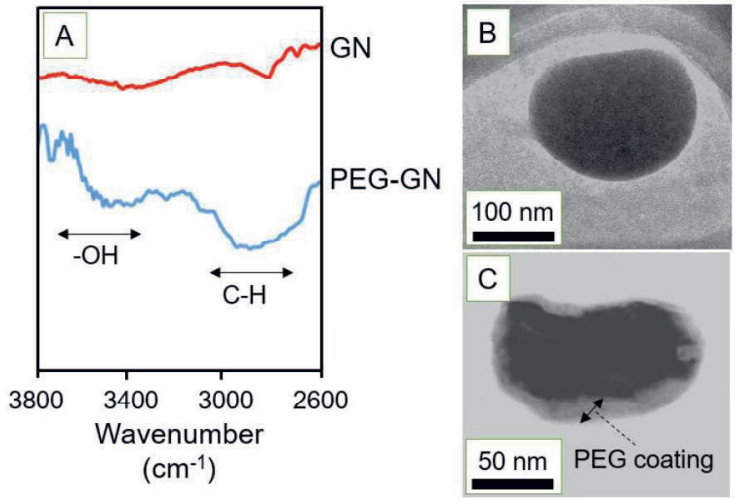

Fig. 1. Characterization of PEG-GN.

mixing, 2-propanol was dropped into the above dispersion to induce coacervation of PEG, during which the coacervated PEG chains were attached to GN through a physical adsorption and PEG-GN were formed. In order to investigate the optimal condition to prepare the PEG-GN possessing size separation function, anti-CRP antibody-FITC or immunocomplex (CRP/anti-CRP antibody-FITC) was mixed with PEG-GN dispersion, and fluorescence images were obtained. Finally, the CRP solution, antiCRP antibody-FITC and PEG-GN were mixed and measured fluorescence intensities.

\section{Results and discussion}

From FT-IR spectra and TEM images, C-H and $\mathrm{O}-\mathrm{H}$ stretching bands that can be assigned to PEG on GN and the PEG coating on GN was confirmed (Fig. 1). By considering the weight ratios of $P E G$ and $G N(P E G / G N)$, the optimal condition was confirmed (Fig. 2A, B). When the fluorescently-labeled antibody was mixed with PEG-GN, the fluorescence intensity was quite low against control condition. When the immunocomplex was mixed with PEG-GN, the fluorescence intensity increased with the increase of $P E G / G N$, and $P E G / G N=4$ was confirmed as optimal condition. By mixing the CRP solution, anti-CRP antibody-FITC and

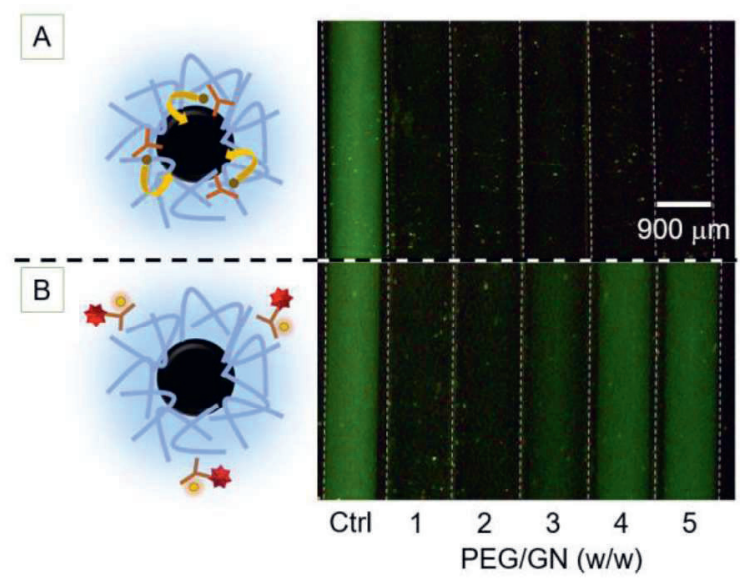

Fig. 2. Investigation of optimal condition to prepare the PEG-GNG possessing size separation function.

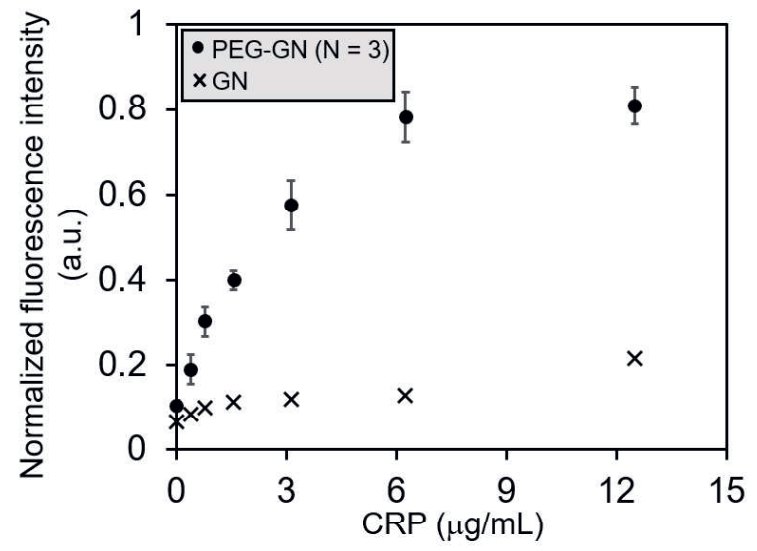

Fig. 3. CRP detection by using PEG-GN.

PEG-GN, fluorescence intensity increased with the increase of CRP concentration (Fig. 3). In the case of using GN instead of PEG-GN, the fluorescence intensity changes were much smaller than the case of using PEG-GN. Thus, the PEG structure was confirmed to play the role of size separation, and the measurement of CRP concentration was achieved.

\section{Conclusions}

It was confirmed that PEG-GN that has size separation function of free fluorescently-labelled antibody and immunocomplex was prepared by coacervation method. Just by mixing PEG-GN, sample solution and anti-CRP antibody-FITC, CRP concentration was successfully measured. In the near future, by integrating this principle into the capillay-type microdevice, the rapid immunosensor will be realized and it will be very useful in the field of POCT.

\section{Acknowledgements}

This work was supported by the Sasakawa Scientific Research Grant from The Japan Science Society, and 2014 Program for Leading graduate schools "Graduate school for Systeminspired Leaders in Material Science". We are greatly acknowledge Mr. Atsuhiro Kotani and Professor Shigeo Mori for TEM measurements.

\section{References}

[1] A. Shirai, T.G. Henares, K. Sueyoshi, T. Endo, H. Hisamoto,

Fast and single-step immunoassay based on fluorescence quenching within a square glass capillary immobilizing graphene oxide-antibody conjugate and fluorescently labelled antibody, Analyst, 141, 3389-3394 (2016); doi: 10.1039/C5AN02637G

[2] A. Shirai, K. Nakashima, K. Sueyoshi, T. Endo, H. Hisamoto, Development of a single-step immunoassay microdevice based on a graphene oxidecontaining hydrogel possessing fluorescence quenching and size separation functions, Analyst, 142, 472-477 (2017); doi: $10.1039 / \mathrm{C} 6 \mathrm{AN} 02485 \mathrm{H}$ 